\title{
Review: assertive community treatment reduced hospital use and was cost effective when used for severely mentally ill patients with previous hospital use of 50 days yearly
}

Latimer EA. Economic impacts of assertive community treatment: a review of the literature. Can J Psychiatry 1999 Jun;44:443-54.

QUESTION: What are the costs associated with assertive community treatment (ACT) when used for the severely mentally ill?

\section{Data sources}

Studies were identified through a literature search, identification of references noted in literature reviews, and communication with experts.

\section{Study selection}

Studies were selected if ACT (community based, multidisciplinary services with shared caseloads) was being evaluated and economic outcomes were reported. Studies were excluded if the programmes investigated were too office based or they did not employ the shared caseload model.

\section{Data extraction}

Data were extracted on percentage difference or reduction in hospital days, the direction and statistical significance of differences in use of various resources (eg, emergency room or outpatient clinic), the study design, degree of fidelity to the ACT model, nature of the control or comparison service, patient characteristics, and duration of follow up.

\section{Main results}

19 randomised controlled trials (RCTs) and 15 non-randomised controlled trials met the selection criteria. High fidelity programmes (those with staff:client ratio of 1:12 or better, a psychiatrist on staff, at least 1 nurse on staff, some coverage outside the normal working hours, and at least 2 team meetings every week) appeared to reduce hospital days by 23 percentage points (95\% CI 5 to 41) more than low fidelity programmes. Regression analysis suggested that high fidelity programmes reduced admissions to hospital by $58 \%$ over 1 year when compared with an alternative that involved some case management and by $78 \%$ when it did not. ACT appeared to increase the proportion of clients living in independent housing, but the effect on use of supervised housing and therefore on housing costs was ambiguous. The effect on use of other resources was inconsistent across studies. Overall, ACT appeared to result in somewhat lower costs. Using Quebec, Canada costs, an ACT programme would have to enrol people with previous hospital use of about 50 days yearly to break even.

For correspondence: Dr E A Latimer, Douglas Hospital Research Centre, 6875 La Salle Boulevard, Verdun, Quebec $\mathrm{H} 4 \mathrm{H}$ 1R3, Canada.Fax +1 5147623049 .

\section{Conclusions}

The most reliable cost effect from assertive community treatment (ACT) was reduced hospital use. Using Quebec, Canada costs, ACT paid for itself when used for severely mentally ill patients with previous hospital use of 50 days yearly.

\section{COMMENTARY}

ACT is now the most extensively studied model of community care for the severely mentally ill. This review by Latimer is the first attempt to review its cost effectiveness and suggests that there may be cost benefits associated with heavy users of inpatient services.

The strengths of this review include the thoroughness in including all relevant studies and the attention to contextual factors. Unfortunately, the author was unable to use the standard instrument to reliably assess model fidelity because of limited reporting on actual service provision in the original studies. Indeed, greater model fidelity was only shown to be associated with reduced hospital admissions when the results from a large Veterans Administration hospitals study was treated as case apart in the regression analysis.

ACT is not a psychiatric treatment: it is a model of how a service can deliver an integrated package of psychosocial rehabilitation and support psychiatric treatment, and we do not know how its effectiveness is mediated. Evidence from the UK suggests that it is not simply a question of caseload size and that substantial decreases in hospital stay only occur when the team has control of the discharge of patients from hospital. ${ }^{1}$ Some evidence exists that the patient-case manager alliance may be important in mediating a favourable response to community care for people with severe mental illness. This raises a question about the importance of not having individual caseloads in the ACT model.

ACT services have been developed with the explicit purpose of reducing hospital admissions or improving contact with difficult to engage patients. Very few studies have measured patient satisfaction, though where this has been done, the outcome has been favourable. A new approach is for service users themselves, with appropriate training and support, to elicit the views of others with severe mental illnesses. ${ }^{2}$ This user focused monitoring may be a valuable tool in evaluating services which attempt to work with difficult to engage patients.

Peter L Cornwall, MB, BS, MRCPsych University of Newcastle, Royal Victoria Infirmary Newcastle Upon Tyne, UK

1 Burns T, Creed F, Fahy T, et al. Intensive versus standard case management for severe psychotic illness: a randomised trial. UK 700 Group. Lancet 1999;353:2185-9.

2 Rose D, Ford R, Lindley P, et al and The KCW Mental Health Monitoring Users' Group. In our experience. User-focused monitoring of mental health services. London: Sainsbury Centre for Mental Health, 1998. 